Pneumologe 2022 $\cdot 19: 42-48$ https://doi.org/10.1007/s10405-021-00427-9

Angenommen: 2. Dezember 2021

Online publiziert: 3 . Januar 2022

(c) Der/die Autor(en) 2022

\section{Zystische Fibrose und Schmerzen - ein unterschätztes Problem}

\author{
Ein narratives Review
}

\author{
Anna Teresa Hoffmann · Stefanie Dillenhöfer · Thomas Lücke · Christoph Maier · \\ Folke Brinkmann \\ Universitätsklinik für Kinder- und Jugendmedizin, Ruhr-Universität Bochum, Bochum, Deutschland
}

\title{
Zusammenfassung
}

Hintergrund: Zystische Fibrose (CF) ist eine der häufigsten vererbbaren Stoffwechselerkrankungen in Deutschland. Sie betrifft verschiedene Organsysteme und führt häufig zu Schmerzen, die meist unterschätzt werden. Im deutschsprachigen Raum existieren bis dato keinerlei Leitlinien zu diesem relevanten Thema.

Methoden: Es erfolgte eine systematische Literaturrecherche in PubMed sowie der deutschsprachigen Leitlinien und Fachliteratur zum Thema CF und Schmerzen. Als relevant wurden Arbeiten gewertet, die Daten zu CF, zu Schmerzen oder deren Behandlung bei der CF enthielten. Insgesamt fanden sich 1690 Artikel, von denen 41 Arbeiten unsererseits aufgenommen wurden.

Ergebnisse: Die Recherche ergab, dass Schmerzen unterschiedlichster Art aufgrund der diversen Organbeteiligungen weit verbreitet sind. Zur Diagnostik und Therapie von Schmerzen bei CF existieren aber keine deutschsprachigen Leitlinien oder Konsenspapiere. In europäischen und amerikanischen Studien spiegelt sich die klinische Relevanz und Bandbreite des Themas wider, Daten zur Effektivität der Schmerztherapie enthalten diese jedoch nicht. Neben einer klassischen Schmerzmedikation mit Analgetika setzen viele Patienten auch auf alternative Heilversuche wie physikalische Therapien, Akupunktur oder Tetrahydrocannabinol (THC). Angesichts der multiplen Organdysfunktionen ist der Einsatz von Analgetika mit besonderen Risiken verbunden.

Diskussion: Eine Schmerztherapie ist bei Patienten mit zystischer Fibrose und Multiorganbeteiligung häufig erforderlich und muss der Progression der Erkrankung angepasst werden. Kontraindikationen und Nebenwirkungen der Analgetika sollten individuell ermittelt werden. Die Aufnahme der Behandlung von Schmerzen in deutschsprachige CF-Leitlinien ist dringend erforderlich.

\section{Schlüsselwörter}

Chronische Erkrankung · Komplikationen · Therapie · Leitlinie · Nebenwirkung

Die beiden Autoren Christoph Maier und Folke Brinkmann teilen sich die Letztautorenschaft

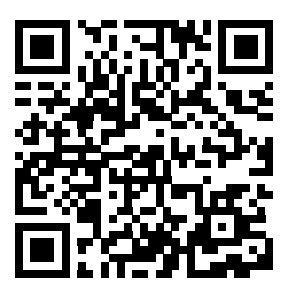

QR-Code scannen \& Beitrag online lesen
Zystische Fibrose (CF) ist eine der häufigsten Stoffwechselerkrankungen in Deutschland. Sie betrifft verschiedene Organsysteme und führt häufig zu Schmerzen, die jedoch im deutschsprachigen Raum in der Regel nicht suffizient erfasst werden; bisher wird vermutlich auch daher das Thema nicht in den Leitlinien zur Behandlung der CF erwähnt.

\section{Hintergrund}

Die zystische Fibrose (CF [MIM 219700]) ist eine progrediente autosomal-rezessiv vererbte Multisystemerkrankung. Neben der meist lebenslimitierenden Progression der Lungenbeteiligung liegen v. a. gastrointestinale Symptome vor. Die Inzidenz in der mitteleuropäischen Bevölkerung liegt bei 1:3500. Für Neugeborene beträgt die Le- 


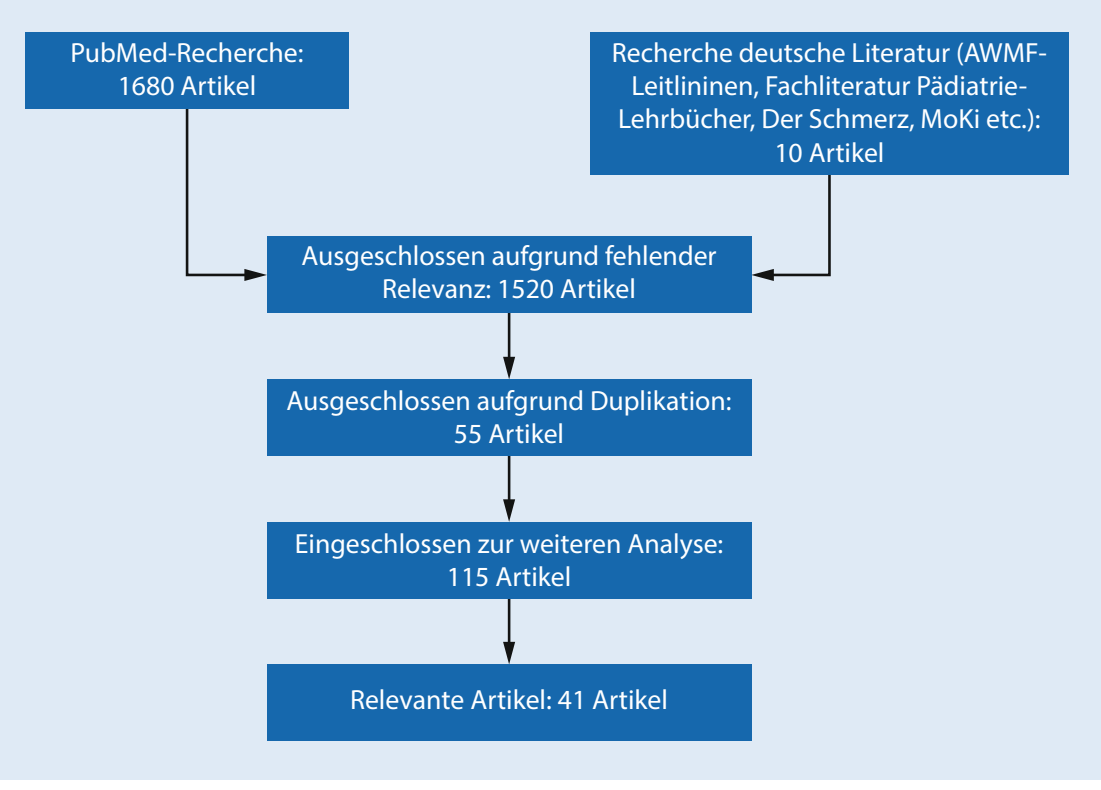

Abb. 1 \ Flowchart eingeschlossenen relevante Artikel

benserwartung aktuell 53 Jahre [29, 32]. Sechs verschiedene Mutationsklassen im CFTR-Gen (langer Arm des Chromosom 7) führen zu gravierenden Funktionsstörungen des CFTR-Chlorid-lonen-Kanals in der Zellmembran. Die daraus resultierende Viskositätserhöhung der Sekrete verschiedener Organsysteme führt zu einer Obstruktion der entsprechenden Hohlräume. Befragungen zeigen, dass die Lebensqualität Betroffener durch eine verminderte Belastbarkeit und Dyspnoe reduziert ist. Häufig finden sind Schmerzen eine Ursache einer reduzierten Lebensqualität $[12,13,16,29$, 32]. Wie wir kürzlich in einem Fallbericht zeigen konnten, gibt es jedoch weder Studien zur Effektivität und noch zu den, wie dort gezeigt, nicht unerheblichen Risiken einer Schmerztherapie [14]. Im deutschsprachigen Raum wurden Schmerzen bei CF als Problem bislang nicht systematisch untersucht. Folgerichtig werden weder die Schmerzsymptomatik noch mögliche Behandlungsoptionen in aktuellen CF-Leitlinien oder sonstigen Leitlinien zur Schmerztherapie aufgegriffen $[1,38]$. Die vorliegende Arbeit soll dafür eine erste Grundlage liefern.

\section{Methoden}

Es erfolgte eine Recherche in PubMed mit den Suchwörtern "cystic fibrosis" AND „pain“ AND „adults" OR „children“, zusätz- lich "treatment" OR „adverse events" AND „opioid". Zudem erfolgte eine Recherche in aktuellen deutschsprachigen AWMF-Leitlinien und Lehrbüchern. Insgesamt fanden sich 1690 Artikel, von denen aufgrund mangelnder Relevanz bzw. Duplikationen 1575 Artikel ausgeschlossen wurden (- Abb. 1); 41 Arbeiten (PubMed-Recherche: Originalarbeiten $[n=23]$, Reviews $[n=7]$, Fallberichte $[n=4]$; deutschsprachige Literatur wie Lehrbücher, AWMFLeitlinien oder Übersichtsarbeiten $[n=7]$ ) wurden aufgenommen, sofern sie Daten zu $\mathrm{CF}$, zu Schmerzen oder deren Behandlung bei der CF enthielten oder aufarbeiten.

\section{Ergebnisse - Stand der Wissenschaft}

In den von uns erfassten Studien, die auch in der aktuellen Übersichtsarbeit von Trandafir et al. enthalten sind [32], wurden 1950 Patienten mit $C F$, 438 Behandler und 100 pflegende Angehörige befragt. Hierzu gehören Studien aus den USA $(n=14)$, Europa $(n=6)$ sowie Kanada $(n=1)$ und Australien $(n=2)$, die größtenteils retrospektiv $(n=20)$ die Schmerzhäufigkeit und -frequenz, ihre Lokalisation und Qualität sowie die Auswirkungen auf das alltägliche Leben in Interviews $(n=4)$ oder durch Fragebögen ( $n=19$ ) erfassten.Zudem fanden wir 2 Beobachtungsstudien sowie eine prospektive Studie. Unsere Recherche ergab keine Studien aus Deutschland. Schmerztherapie bei $\mathrm{CF}$ als Stichwort fand sich auch nicht in deutschen Lehrbüchern oder Leitlinien. Einige wenige nichtmedikamentöse Therapieoptionen wie Yoga und Osteopathie wurden auch prospektiv geprüft (s. unten) $[15,20]$.

Die Lokalisation von Schmerzen bei der CF variiert stark entsprechend dem Ausmaß der jeweiligen Organaffektion (ब Tab. 1). Hierin unterscheiden sich Kinder und Jugendliche von Erwachsenen. Erstere haben zu nahezu $100 \%$ an $1 \mathrm{Tag}$ pro Monat akute abdominelle Schmerzen $[16,29,32]$, dagegen nur bis zu $50 \%$ der Erwachsenen [32]. Kinder und Jugendliche klagen eher über intermittierend bzw. rezidivierende Schmerzen mit längeren Phasen der Schmerzfreiheit $[12,16,29$, 32]. Sobald pulmonale Exazerbationen mit Symptomen wie Dyspnoe, vermehrtem Husten, zähem Sputum, Gewichtsabnahme und Müdigkeit zunehmen, treten Thoraxschmerzen in den Vordergrund $[12,29,32]$. Sie sind daher mit der Progression der Lungenerkrankung und einer reduzierten Einsekundenkapazität $\left(\mathrm{FEV}_{1}\right)$ und schlechteren Atemwegs-Clearance eng verbunden [32]. Die Frequenz dieser Episoden steigt ab dem Jugend- bis in das Erwachsenenalter zunehmend an und nimmt in den letzten 3 Lebensmonaten deutlich zu. Thoraxschmerzen gehen somit mit einer erhöhten Mortalität einher $[12,13,32]$, wie Hayes et al. [13] in ihrer Studie zeigen konnten.

Erst im Erwachsenenalter kommt es häufiger auch zu chronischen Schmerzen, d.h. schmerzfreie Episoden werden kaum noch berichtet, da neben den Thoraxschmerzen auch Rücken-, Kopf- und Nacken- und Gelenkschmerzen auftreten ( $\mathbf{0}$ Tab. 1; [12, 13, 32]). Insbesondere Thoraxschmerzen gehen mit einer reduzierten Teilhabe am alltäglichen Leben sowie Depressionen und Angststörungen einher [25, 32]. Die Prävalenz von Rückenund Kopfschmerzen wird von einigen Autoren höher als in der Allgemeinbevölkerung eingeschätzt $[12,26,27,32]$. Ein Vergleich mit der Allgemeinbevölkerung ist u. E. schwierig, da in den vorliegenden Studien die Stichtagsprävalenz bzw. lediglich Schmerzen der letzten 6 Monate erhoben wurden und die Altersgruppe der Erwachsenen nicht vergleichbar ist 


\begin{tabular}{|c|c|c|c|}
\hline Lokalisation & $\begin{array}{l}\text { Kinder \& Ju- } \\
\text { gendliche (\%) }\end{array}$ & $\begin{array}{l}\text { Erwachsene } \\
(\%)\end{array}$ & Pathophysiologie/häufige Ursachen \\
\hline \multirow[t]{6}{*}{$\begin{array}{l}\text { Abdominelle } \\
\text { Schmerzen }\end{array}$} & \multirow[t]{6}{*}{$42-100$} & \multirow[t]{6}{*}{$19-50$} & $\begin{array}{l}\text { Distales intestinales Obstruktionssyndrom } \\
\text { (DIOS) mit Komplikation wie Subileus, lleus } \\
\text { oder Invagination }\end{array}$ \\
\hline & & & Pankreatitis \\
\hline & & & Unterdosierung Pankreasenzyme \\
\hline & & & $\begin{array}{l}\text { Hepatobiliäre Komplikationen wie Gallen- } \\
\text { steine oder Entzündungen }\end{array}$ \\
\hline & & & $\begin{array}{l}\text { Nicht CF-spezifische GI-Erkrankungen wie } \\
\text { Enteritis, Appendizitis }\end{array}$ \\
\hline & & & $\begin{array}{l}\text { Extraabdominale Ursachen: Exazerbation, } \\
\text { Pneumonie }\end{array}$ \\
\hline \multirow{3}{*}{$\begin{array}{l}\text { Obere Abdo- } \\
\text { minalschmer- } \\
\text { zen }\end{array}$} & \multirow[t]{3}{*}{10} & \multirow[t]{3}{*}{$10-51$} & Gastrointestinale Ulzera \\
\hline & & & Gastroösophagealer Reflux \\
\hline & & & Gastritis \\
\hline \multirow{6}{*}{$\begin{array}{l}\text { Thorax- } \\
\text { schmerzen }\end{array}$} & \multirow[t]{6}{*}{$10-38$} & \multirow[t]{6}{*}{$9-72$} & Exazerbation \\
\hline & & & Pneumonie \\
\hline & & & $\begin{array}{l}\text { Komplikationen wie Pleuritis oder Pneumo- } \\
\text { thorax }\end{array}$ \\
\hline & & & Rippenfrakturen \\
\hline & & & Muskuläre Dysfunktion \\
\hline & & & $\begin{array}{l}\text { Schmerzhafte Interventionen (Spirometrie, } \\
\text { Bronchoskopie, autogene Drainage) }\end{array}$ \\
\hline \multirow[t]{2}{*}{$\begin{array}{l}\text { Rücken- } \\
\text { schmerzen }\end{array}$} & \multirow[t]{2}{*}{$6-16$} & \multirow[t]{2}{*}{$19-70$} & $\begin{array}{l}\text { Komplikationen der Osteoporose mit Ky- } \\
\text { phosen; Frakturen }\end{array}$ \\
\hline & & & Muskuläre Dysfunktion \\
\hline \multirow{3}{*}{$\begin{array}{l}\text { Kopf- und } \\
\text { Nacken- } \\
\text { schmerzen }\end{array}$} & \multirow[t]{3}{*}{$13-42$} & \multirow[t]{3}{*}{$6-64$} & Sinusitiden \\
\hline & & & Nasale Polyposis \\
\hline & & & Siehe Rückenschmerzen \\
\hline \multirow{2}{*}{$\begin{array}{l}\text { Gelenk- } \\
\text { schmerzen }\end{array}$} & \multirow[t]{2}{*}{$11-19$} & \multirow[t]{2}{*}{$6-27$} & Arthritis \\
\hline & & & $\begin{array}{l}\text { Hypertrophische pulmonale Osteoarthropa- } \\
\text { thie }\end{array}$ \\
\hline Myalgien & $3-19$ & $6-44$ & Inaktivität \\
\hline
\end{tabular}

(Erwachsene mit CF im Mittel zwischen 23,5 und 31 Jahren) [32].

Neben Schmerzen durch die Grunderkrankung selbst klagen viele Betroffene zudem über iatrogene Schmerzen bei Untersuchungen wie Blutentnahmen und Lungenfunktionsuntersuchungen, aber auch im Rahmen ihrer symptomatischen Therapien, insbesondere bei der autogenen Drainage zur Sekretmobilisation und Atemphysiotherapie [8, 29]. Durch Letztere kommt es häufig zu einer NonCompliance bei der Durchführung dieser täglich notwendigen Therapie und damit zur Begünstigung von Exazerbationen [13].

Die Thorax- und Abdominalschmerzen insbesondere der Kinder und Jugendlichen sind gemäß der bald aktuellen ICD-11-Klas- sifikation [33] als sekundär chronische viszerale rezidivierende Schmerzen einzuordnen mit unterschiedlich langen schmerzfreien Phasen [12, 16, 29, 32], d.h. es sind Schmerzen, die zwar lange, oft sogar lebenslang bestehen, bei denen es aber v. a. bei den Kindern und Jugendlichen regelhaft auch längere Remissionszeiten ohne Schmerzen gibt [19]. In der Häufigkeit und der Wiederholungsrate von Schmerzepisoden bestehen keine wesentlichen Unterschiede zwischen Erwachsenen und Kindern [29], jedoch sind die Schmerzepisoden bei Erwachsenen länger und gehen mit höheren Schmerzleveln einher [12, 16, 29, 32]. Laut Sermet-Gaudelus et al. [29] leiden $73 \%$ der Erwachsenen an chronischen Schmerzen, die in dieser Studie als Schmerzen definiert sind, die seit mehr als 6 Monaten anhalten oder rekurrieren [29]. Hier ist insbesondere bei Kindern, die öfter an intermittierenden Schmerzen leiden, eine verständliche Aufklärung der Patienten und Eltern über Verlauf, konkrete Therapiemöglichkeiten im Akutfall sowie das sinnvolle Absetzen von Analgetika in schmerzfreien Phasen erforderlich.

Ein gravierendes Problem scheint nach dem Schrifttum und auch nach eigenen Erfahrungen das geringe Problembewusstsein der Behandler für Schmerzen bei CFPatienten. Viele Betroffene verschweigen, da nicht befragt, ihre Schmerzen, und greifen zu Selbstmedikation mit freiverkäuflichen Analgetika [29, 32, 37]. Hier kommen neben Paracetamol (59\%) hauptsächlich nichtsteroidale Antirheumatika (NSAR; $10 \%)$, Acetylsalicylsäure (5\%) und Spasmolytika zum Einsatz [29]. Bei stärkeren Schmerzen setzen Behandler neben den oben genannten Analgetika weitere Substanzklassen ein. Opioide werden v. a. in der Palliativbehandlung zur Symptomkontrolle von Schmerzen und Dyspnoe eingesetzt, aber auch als Mittel der letzten Wahl bei Episoden mit starken Schmerzen $[9,37]$. Auch Gabapentin kommt in amerikanischen CF-Zentren insbesondere bei Thoraxschmerzen zum Einsatz [37]. In Einzelfällen werden auch immer wieder invasive Therapien zur Behandlung abdomineller und thorakaler Schmerzen wie die Epiduralanalgesie mittels Kathetern und die intrapleurale Injektion von Fentanyl oder Bupivacain eingesetzt [5, 7]. Über die Wirksamkeit der eingesetzten Analgetika liegen ebenso wie zu den spezifischen Risiken der Therapie bei CF keine Daten vor $[12,13,16,29,32,37]$.

Im Jahr 2020 erfolgte eine Befragung in den USA zum Konsum von medizinischen sowie generell von Tetrahydrocannabinol(THC)-haltigen Substanzen bei CF-Patienten im Alter ab 12 Jahren mit der größten Gruppe zwischen 21 und 34 Jahren [30]. Von $16,5 \%$, die den Konsum von THC-haltigen Substanzen zu medizinischen Zwecken (Entspannung bei Angstsymptomatik, Behandlung von hauptsächlich muskuloskeletalen Schmerzen, Appetitstimulation) angaben, verspürten $90,3 \%$ einen guten bis sehr guten Effekt [30]. Offenbleiben muss jedoch, ob hier nicht gewollt psychische Effekte im Vordergrund standen. Bekanntlich ist der Effekt von THC 
auf akute Schmerzen derartig gering, dass seine Anwendung hierbei von den Fachgesellschaften ausdrücklich nicht empfohlen wird [10, 24].

Zusätzlich versuchen sich einige Patienten in alternativen Strategien und Therapieansätzen, die von Homöopathie über Akupunktur bis zu physikalischen Maßnahmen (Ruhe oder Aktivität, Wärme oder Kälte, Yoga und Meditation, Osteopathie) reichen [15, 20, 30, 32]. Hier existieren wenige prospektive Studien zu Wirksamkeit und Effektivität. In einer prospektiven, aber nicht kontrollierten Studie wurden Gelenkschmerzen und Ängste durch eine regelmäßige Yogatherapie vermindert [20]. Eine qualitativ hochwertige dreiarmige RCT mit aber nur 32 Probanden zeigte, dass eine osteopathische Behandlung $z u$ einem Rückgang von Rücken- und Thoraxschmerzen führt, jedoch erbrachte die osteopathische Behandlung gegenüber den 2 Kontrollgruppen (Gruppe 2: Scheinbehandlung [Körperkontakt ohne Intervention], Gruppe 3: keine zusätzliche Intervention neben der normalen Therapie) keine signifikante Besserung [15].

\section{Diskussion}

Schmerzen von Patienten mit CF sind nachweislich ein häufiges und für die Betroffenen relevantes, jedoch in Deutschland bislang wenig diskutiertes Problem, das sich in den aktuellen CF-Leitlinien nicht widerspiegelt [1]. Betroffen sind alle Altersklassen $a b$ dem frühen Kindesalter [16, 29, 32]. Die Häufigkeit und Intensität von Schmerzen bei CF erklären sich zum Teil durch die typischen Komplikationen der Erkrankung wie Thoraxschmerzen bei Exazerbationen, Pleuritiden oder Osteoporose-bedingten Rippenfrakturen oder aber als therapiebegleitendes Symptom (- Tab. 1). Eine akute Obstruktionssymptomatik wie beim distalen intestinalen Obstruktionssyndrom (DIOS) verursacht häufig abdominelle Schmerzen [18, 22]. Die erschwerte Schmerzkontrolle bei CF könnte zudem durch eine generelle Senkung der Schmerzschwellen durch rezidivierende Ausschüttung proinflammatorischer Zytokine bedingt sein [32]. Auch könnte die der CF zugrunde liegende Fehlfunktion der CFTR-Kanäle die körpereigene Schmerzhemmung über
Neuronen des peripheren und zentralen Nervensystems negativ beeinflussen. Die Aktivierung von Chloridkanälen führt hier zu einer Depolarisation, die abhängig vom Chloridgleichgewicht und der Geschwindigkeit des Chloridstroms sowohl eine Inhibition als auch eine Exzitation hervorrufen kann [36].

Unsere Literaturrecherche zeigt die vielfältigen Herausforderungen bei der Schmerztherapie von Kindern, Jugendlichen oder auch Erwachsenen mit CF auf. Zu beachten ist hier, dass sich nicht nur die Schmerzarten, sondern auch die Schmerzdauer mit zunehmendem Alter verändert bzw. die Häufigkeit schmerzfreier Intervalle abnimmt (•Tab. 1).

(i) Bereits ab dem frühen Kindesalter treten meist zwar heftige rezidivierende Schmerzepisoden auf, dazwischen aber unterschiedlich lange schmerzfreien Phasen [29]. Es müssen daher Medikamente mit niedrigem Abhängigkeitspotenzial Verwendung finden, die rasch auch wieder abgesetzt werden können. Außerdem sollten Konzepte zur Behandlung von Akutschmerzen mittels Bedarfsmedikation im häuslichen Setting mit den Familien besprochen werden. Auch therapiebegleitende Schmerzen müssen adäquat erfasst und behandelt werden, um Komplikationen wie Exazerbationen durch Non-Compliance zu vermeiden und die Teilhabe am alltäglichen Leben zu verbessern [13, 16, 32]. Bei Jugendlichen treten meist heftige rezidivierende Schmerzen auf, die schmerzfreien Phasen sind häufig kürzer als im frühen Kindesalter und Schmerzen können in Einzelfällen auch schon chronisch auftreten [13, 16, 32]. Bei Erwachsenen mit CF treten mit Progression der Erkrankung dann typischerweise chronische Schmerzen auf [29], hier kann in schweren Fällen eine Dauermedikation indiziert sein. Die Schmerzlast liegt bei CFPatienten dabei deutlich über dem Niveau der Allgemeinbevölkerung [12, 26, 27, 32].

(ii) CF ist eine Systemerkrankung mit einer großen Bandbreite im Ausmaß der Organbeteiligungen (• Tab. 1). Hieraus ergeben sich neben den unterschiedlichen Therapieanforderungen bei akuten und chronischen Schmerzen spezifische Kontraindikationen für einzelne Analgetika

(- Tab. 2). Ein starres Schema verbietet sich somit, da Analgetika in bestimmten Konstellationen Mittel der 1. Wahl, in anderen dagegen kontraindiziert sein können. Auch nichtmedikamentöse Therapiemaßnahmen wie Sport, Yoga etc. sollten in die Behandlung miteinbezogen werden.

(iii) Bisher existieren keine krankheitsspezifischen Leitlinien oder Daten zur analgetischen Wirksamkeit sowie insbesondere vergleichende Studien fehlen vollständig, sodass aktuell auf die allgemeinen Leitlinien zum Gebrauch von Opioiden und Nichtopioiden bei der Schmerztherapie zurückgegriffen werden muss [19]. Es existieren lediglich Daten zur Häufigkeit des Einsatzes diverser Analgetika, nicht selten auf der Basis der Selbstmedikation. Hier besteht somit erheblicher Forschungsbedarf. Zudem gibt es für bestimmte in der Akutschmerztherapie sonst bewährte Substanzen wie Metamizol oder Coxibe bislang keine Untersuchungen. Auch für bestimmte Opioide wie Buprenorphin, welches aufgrund seines günstigen Nebenwirkungsprofils Vorteile gegenüber anderen Opioiden haben könnte, wie von uns kürzlich in einer Kasuistik gezeigt [14], fehlen entsprechende Daten. Das Risiko einer Überdosierung und auch Fehlmedikation u. a. durch Nichterfassung einer Selbstmedikation ist hoch. Eine Erhöhung der Sensibilität für dieses Thema ist somit dringend erforderlich. Es sollte mehr als bisher Bestandteil der Aufklärung von Eltern und Patienten sein.

Gängige Analgetika wie Paracetamol, NSAR, Acetylsalicylsäure, Gabapentin und Opioide sollten in kommenden Studien auch auf ihre Nebenwirkungen überprüft werden. Hierbei erschwert häufig v. a. eine reduzierte Leber- und teilweise auch Nierenfunktion die Auswahl und Dosierung der Analgetika. Bei der Auswahl eines geeigneten Nichtopioids sollten bei 
Tab. 2 Potenzielle Analgetika in der Schmerztherapie bei CF; Dosisempfehlung adaptiert nach [35]

\begin{tabular}{|c|c|c|c|}
\hline Analgetikum & Dosierung und Zulassung & Potenzielle Risiken & Potenzielle Anwendungsgebiete \\
\hline $\begin{array}{l}\text { Paracetamol } \\
\text { p.o., supp, i.v. }\end{array}$ & $\begin{array}{l}10-15 \mathrm{mg} / \mathrm{kg} / \mathrm{ED} \\
\text { Max. } 60 \mathrm{mg} / \mathrm{kg} / \mathrm{Tag} \text { bzw. } 4 \mathrm{~g} / \mathrm{Tag} \\
\text { Ab Geburt }\end{array}$ & $\begin{array}{l}\text { Hepatotoxizität, Übelkeit, Bauch- und Kopfschmer- } \\
\text { zen }[2,19,35] \\
\text { cave: CF-Hepatopathie, Leberzirrhose }\end{array}$ & $\begin{array}{l}\text { Ggf. Thoraxschmerzen, ggf. viszerale/ } \\
\text { abdominelle Schmerzen, therapiebeding- } \\
\text { te Schmerzen [32] }\end{array}$ \\
\hline $\begin{array}{l}\text { Ibuprofen p.o., } \\
\text { supp }\end{array}$ & $\begin{array}{l}10 \mathrm{mg} / \mathrm{kg} / \mathrm{ED} \\
\text { Max. } 40 \mathrm{mg} / \mathrm{kg} / \mathrm{Tag} \text { bzw. } \\
2,4 \mathrm{~g} / \mathrm{Tag} \\
\geq 3 \text { Monate bzw. } \geq 5 \mathrm{~kg}\end{array}$ & $\begin{array}{l}\text { GI-Ulzera/-Blutung, Nephrotoxizität } \\
\text { Erhöht Inflammation in niedriger Dosierung }[2,12 \text {, } \\
13,19,32,35] \\
\text { Cave: Hyperazidität, gastroösophagealer Reflux, } \\
\text { sekundäre Nephropathie }[11,19,23,31]\end{array}$ & $\begin{array}{l}\text { Kopfschmerzen, Arthritiden (z. T. mit Kor- } \\
\text { tison) [21], muskuloskeletale Schmerzen } \\
\text { [32], ggf. viszerale/abdominelle Schmer- } \\
\text { zen, therapiebedingte Schmerzen [32] }\end{array}$ \\
\hline $\begin{array}{l}\text { Metamizol } \\
\text { p.o., i.v. }\end{array}$ & $\begin{array}{l}10-15 \mathrm{mg} / \mathrm{kg} / \mathrm{ED} \\
\text { Max. } 75 \mathrm{mg} / \mathrm{kg} / \mathrm{Tag} \text { bzw. } 5 \mathrm{~g} / \mathrm{Tag} \\
\geq 3 \text { Monate bzw. } \geq 5 \mathrm{~kg}\end{array}$ & $\begin{array}{l}\text { Hypotension bei rascher i.v.-Gabe, Agranulozytose } \\
\text { (regelmäßige Blutbildkontrollen notwendig) [17, } \\
\text { 35] }\end{array}$ & Viszerale/abdominelle Schmerzen [32] \\
\hline $\begin{array}{l}\text { Acetylsalicyl- } \\
\text { säure } \\
\text { p.o. }\end{array}$ & $\begin{array}{l}\text { Analgesie: } 10-15 \mathrm{mg} / \mathrm{kg} / \mathrm{ED} \\
\text { Max. } 60-80 \mathrm{mg} / \mathrm{kg} / \mathrm{Tag} \text { bzw. } \\
3 \mathrm{~g} / \mathrm{Tag} \\
\geq 12 \text { Jahre }\end{array}$ & $\begin{array}{l}\text { Erhöhte Blutungsneigung, Übelkeit, Erbrechen, } \\
\text { Unruhe, Reye-Syndrom [35] }\end{array}$ & $\begin{array}{l}\text { Muskuloskeletale Schmerzen, Kopf- } \\
\text { schmerzen }\end{array}$ \\
\hline $\begin{array}{l}\text { Parecoxib i.v., } \\
\text { i.m. }\end{array}$ & $\begin{array}{l}40 \mathrm{mg} \text { als ED, max. } 80 \mathrm{mg} / \mathrm{Tag} \\
\geq 18 \text { Jahre }\end{array}$ & $\begin{array}{l}\text { Seltener gastrointestinale Nebenwirkungen als } \\
\text { lbuprofen, Nephrotoxizität, Dosisreduktion bei } \\
\text { Leberfunktionsstörung und Gewicht }<50 \text { kg erfor- } \\
\text { derlich }[2,19,35]\end{array}$ & Siehe Ibuprofen \\
\hline $\begin{array}{l}\text { Butylscopol- } \\
\text { amin p.o., } \\
\text { supp, i.v. }\end{array}$ & $\begin{array}{l}0,3-0,6 \mathrm{mg} / \mathrm{kg} / \mathrm{ED} \\
\text { Max. 1,5 mg/kg/Tag bzw. Kinder } \\
60 \mathrm{mg} / \mathrm{Tag} ; \text { Erw. } 100 \mathrm{mg} / \mathrm{Tag} \\
\geq 6 \text { Jahre }\end{array}$ & $\begin{array}{l}\text { Tachykardie, Obstipation, Miktions- und Akkom- } \\
\text { modationsstörung [35] }\end{array}$ & $\begin{array}{l}\text { Viszerale/abdominelle Schmerzen (mit } \\
\text { Metamizol) [32] }\end{array}$ \\
\hline $\begin{array}{l}\text { Gabapentin } \\
\text { p.o. }\end{array}$ & $\begin{array}{l}\text { Neuropathische Schmerzen: } \\
\text { Zieldosis 3-mal } 15-30 \mathrm{mg} / \mathrm{kg} / \mathrm{ED} \\
\text { (Aufdosieren über } 3 \text { bis } 7 \text { Tage) } \\
\text { Max. 3,6 } 6 \text { /Tag } \\
\geq 18 \text { Jahre }\end{array}$ & $\begin{array}{l}\text { Schwindel, Kopfschmerzen, Benommenheit, Paräs- } \\
\text { thesien, emotionale Instabilität, Obstipation, Dys- } \\
\text { pepsie [35] } \\
\text { Cave: Depression, Angststörung, sozialer Rückzug } \\
\text { [25, 32], DIOS }\end{array}$ & $\begin{array}{l}\text { Thoraxschmerzen („off-label“) [37], neu- } \\
\text { ropathische Schmerzen }\end{array}$ \\
\hline Piritramid i.v. & $\begin{array}{l}0,05-01 \mathrm{mg} / \mathrm{kg} / \mathrm{ED} \text { alle } 4-6 \mathrm{~h} \\
\geq 6 \text { Monate }\end{array}$ & \multirow{2}{*}{$\begin{array}{l}\text { Atemdepression, Hypoxie (ggf. stationäre Über- } \\
\text { wachung bei Therapieeinleitung erforderlich), } \\
\text { Bronchospasmus, Juckreiz, Flush, Hypotension, } \\
\text { Bradykardie, Obstipation } \\
{[4,9,12,28,32,34,35,37]} \\
\text { Cave: fortgeschrittene Lungenbeteiligung; DIOS }\end{array}$} & \multirow{2}{*}{$\begin{array}{l}\text { Thoraxschmerzen [37], hypertrophische } \\
\text { pulmonale Osteoarthropathie [21], Pallia- } \\
\text { tivmedizin [9] }\end{array}$} \\
\hline $\begin{array}{l}\text { Buprenorphin } \\
\text { s.l. }\end{array}$ & $\begin{array}{l}0,2-0,4 \mathrm{mg} / \mathrm{ED} \text { alle } 6-8 \mathrm{~h} \\
\geq 15 \text { Jahre }\end{array}$ & & \\
\hline $\begin{array}{l}\text { Medizin. Ma- } \\
\text { rihuana (THC) }\end{array}$ & Keine Empfehlung vorliegend & $\begin{array}{l}\text { Chronischer Husten, erhöhte Sputumprodukti- } \\
\text { on, vermehrtes Giemen, Kurzatmigkeit; akute } \\
\text { Bronchitis, Müdigkeit, Schwindel, Erbrechen, Hal- } \\
\text { luzinationen [30] } \\
\text { Cave: fortgeschrittene Lungenbeteiligung }\end{array}$ & $\begin{array}{l}\text { (Muskuloskeletale) Schmerzen, Angstzu- } \\
\text { stände, Appetitlosigkeit, Schlafstörungen } \\
{[30]}\end{array}$ \\
\hline
\end{tabular}

erwünschter antiinflammatorischer Wirkung NSARs oder Coxibe gewählt werden, ansonsten Paracetamol oder Metamizol, wobei stets das individuelle Risikoprofil beachtet werden muss [19]. Hauptgefahren von NSAR sind die erhöhte Nephrotoxizität, v. a. in Kombination mit den häufig bei Exazerbationen eingesetzten Aminoglykosiden, sowie die gastrointestinale Blutungsgefahr bei bekannter CFassoziierter Hyperazidität [11, 19, 23, 31]. Bei einer CF-bedingten Hepatopathie ist an eine Dosisreduktion oder den Verzicht von Paracetamol aufgrund seiner Hepatotoxizität zu denken [6].

Hinzu kommen die opioidvermittelten Risiken, die wir bereits mittels eines kürzlich veröffentlichten Fallberichts eines Patienten mit stärksten Thoraxschmerzen [14] zeigen konnten. Die Intensität der Thoraxschmerzen korreliert mit der Progression der Erkrankung [13, 32]. Schmerzen vermindern zusätzlich die Atemexkursion und erschweren die Drainagetherapie. Aber auch die dann oft unverzichtbaren Opioide können bei falscher Auswahl und zu hoher Dosierung nächtliche Hypoxie verstärken und dosisabhängig zu Atemdepression führen [4, 9, 12, 32, 37]. Dosisunabhängig können Opioide durch die Verminderung der Darmmobilität über eine opioidinduzierte Obstipation (OIC) die Gefahr eines distalen Obstruktionssyndroms (DIOS) und Subileus erhöhen
[28, 34]. Die Therapieüberwachung sollte im Regelfall durch den behandelnden Pädiater oder Pneumologen erfolgen, in komplizierten Fällen wie bei psychischer Komorbidität oder Hinweisen auf Abhängigkeit sollten Psychologen, Psychiater oder Schmerzmediziner jedoch hinzugezogen werden.

(iv) CF-Patienten werden aufgrund der Versorgungsstrukturen in Deutschland häufig von pädiatrischen Pneumologen auch im Erwachsenenalter weiter behandelt. Neben einer geringen Expertise in der Behandlung chronischer Schmerzen in der Pädiatrie wird zudem bei Kindern und teilweise auch Jugendlichen die 
Auswahl des richtigen Medikaments durch die fehlende Zulassung erschwert (•Tab. 2). Auch bestehen neben dem Mangel an Leitlinien deutliche Versorgungslücken in der Schmerztherapie in Deutschland für Kinder- und Jugendliche mit nur wenigen multidisziplinären Behandlungszentren [38].

Prospektive Studien zur Erfassung des aktuellen Standes der Schmerztherapie in Deutschland bei CF-Patienten sind notwendig, um eine entsprechende Leitlinie unter Beachtung aller Faktoren wie Wirksamkeit, Risiken und Nebenwirkungen ausarbeiten zu können. Zur besseren Vergleichbarkeit sollten standardisierte Fragebögen, die auch CF-spezifische Probleme erfassen, erarbeitet werden und zum Einsatz kommen.

\section{Fazit}

- Eine Schmerzbehandlung ist bei den meisten Patienten mit Zystrischer Fibrose (CF) notwendig und soll der Progression der Erkrankung angepasst werden. Während Kinder meist nur eine kurzzeitige Akuttherapie benötigen, ist bei Erwachsenen häufiger die Therapie chronischer Schmerzen erforderlich. Dabei sollten Medikamente mit geringstem Abhängigkeitspotenzial gewählt werden.

- Bei starken Akutschmerzen soll eine Bedarfsmedikation geprüft werden. Anwendung und Gefahren müssen mit den Patienten bzw. Eltern besprochen werden.

- Die analgetische Effektivität jeder Schmerztherapie (inklusive einer Selbstmedikation) ist zu dokumentieren. Nicht wirksame Medikamente sind abzusetzen.

- Eine rationale Schmerztherapie sollte der unterschiedlichen Ätiologie der Schmerzen Rechnung tragen. Nichtopioide sind bei den meisten Schmerzarten Mittel der 1. Wahl, aber nur, wenn sie ausreichend wirksam und im Einzelfall nicht kontraindiziert sind (- Tab. 2). Opioide sind Medikamente der 2 . oder 3 . Wahl, aber sollten auch dann in der niedrigsten, eben ausreichend wirksamen Dosis eingesetzt werden. Bei komplexen Fällen sollten Psychologen oder Schmerzmediziner in die Therapie involviert werden.

- Obwohl hierzu nur wenige Studien vorliegen, halten wir angesichts der Risiken jeder medikamentösen Therapie die Integration nichtpharmakologischer Verfahren in die Standardtherapie für sinnvoll.

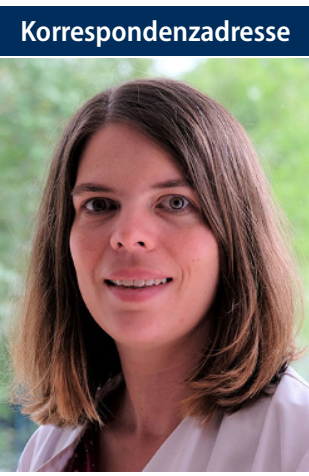

Anna Teresa Hoffmann

Universitätsklinik für Kinder- und Jugendmedizin, Ruhr-Universität Bochum

Alexandrinenstraße 5, 44971 Bochum,

Deutschland

annateresa.hoffmann@klinikum-bochum.de

Funding. Open Access funding enabled and organized by Projekt DEAL.

\section{Einhaltung ethischer Richtlinien}

Interessenkonflikt. A.T. Hoffmann, S. Dillenhöfer, T. Lücke, C. Maier und F. Brinkmann geben an, dass kein Interessenkonflikt besteht.

Für diesen Beitrag wurden von den Autoren keine Studien an Menschen oder Tieren durchgeführt. Für die aufgeführten Studien gelten die jeweils dort angegebenen ethischen Richtlinien.

Open Access. Dieser Artikel wird unter der Creative Commons Namensnennung 4.0 International Lizenz veröffentlicht, welche die Nutzung, Vervielfältigung, Bearbeitung, Verbreitung und Wiedergabe in jeglichem Medium und Format erlaubt, sofern Sie den/die ursprünglichen Autor(en) und die Quelle ordnungsgemäß nennen, einen Link zur Creative Commons Lizenz beifügen und angeben, ob Änderungen vorgenommen wurden.

Die in diesem Artikel enthaltenen Bilder und sonstiges Drittmaterial unterliegen ebenfalls der genannten Creative Commons Lizenz, sofern sich aus der Abbildungslegende nichts anderes ergibt. Sofern das betreffende Material nicht unter der genannten Creative Commons Lizenz steht und die betreffende Handlung nicht nach gesetzlichen Vorschriften erlaubt ist, ist für die oben aufgeführten Weiterverwendungen des $\mathrm{Ma}$ terials die Einwilligung des jeweiligen Rechteinhabers einzuholen.

Weitere Details zur Lizenz entnehmen Sie bitte der Lizenzinformation auf http://creativecommons.org/ licenses/by/4.0/deed.de.

\section{Literatur}

1. AWMF-S3-Leitlinie (2013) Lungenerkrankung bei Mukoviszidose - Modul 1 und 2. https:// www.awmf.org/leitlinien/detail/l//026-022.html.
Zugegriffen: 18. Febr. 2020 (https://www.awmf. org/leitlinien/detail/l//020-018.html)

2. Bolten WW, Krüger Ketal (2016) DGRh-Empfehlungen zur Implementierung aktueller Sicherheitsaspekte in die NSAR-Therapie muskuloskelettaler Schmerzen.ZRheumatol 75(1):103-116

3. Botton E, Saraux A et al (2003) Musculoskeletal manifestations in cystic fibrosis. Joint Bone Spine 70(5):327-335

4. Brown KA, Laferrière $A$ et al (2006) Recurrent hypoxemia in children is associated with increased analgesic sensitivity to opiates. Anesthesiology 105(4):665-669

5. Bruce DL, Gerken MV, Lyon GD (1987) Postcholecystectomy pain relief by intrapleural bupivacaine in patients with cystic fibrosis. Anesth Analg 66(11):1187-1189

6. Brune K, Hinz B, Otterness I (2009) Aspirin and acetaminophen: should they be available over the counter? Curr Rheumatol Rep 11(1):36-40

7. Cain JC, Lish MC, Passannante AN (1994) Epidural fentanyl in a cystic fibrosis patient with pleuritic chest pain. Anesth Analg 78(4):793-794

8. Casier A, Goubert Let al (2013) Spirometry-related pain and distress in adolescents and young adults with cystic fibrosis: the role of acceptance. Pain Res Manag 18(6):286-292

9. Dhingra $L$, Walker $P$ et al (2020) Addressing the burden of illness in adults with cystic fibrosis with screening and triage: An early intervention model of palliative care. J Cyst Fibros 19(2):262-270

10. Fitzcharles MA, ShirY,HäuserW (2019) Medicalcannabis: strengthening evidence in the face of hype and public pressure. CMAJ 19;191(33):E907-E908

11. Grimaldi-Bensouda L, Abenhaim L et al (2010) Clinical features and risk factors for upper gastrointestinal bleeding in children: a case-crossover study. Eur J Clin Pharmacol 66(8):831-837

12. Havermans T, Colpaert K et al (2013) Pain in CF: review of the literature. J Cyst Fibros 12(5):423-430

13. Hayes M, Yaster M et al (2011) Pain is a common problem affecting clinical outcomes in adults with cystic fibrosis. Chest 140(6):1598-1603

14. Hoffmann AT, Maier C et al (2021) Herausforderungen der Therapie thorakaler Schmerzen bei zystischer Fibrose (CF) [The challenges of managing thoracic pain in cystic fibrosis (CF)]. Schmerz. https://doi.org/10.1007/s00482-021-00603-z

15. Hubert D, Soubeiran L et al (2014) Impact of osteopathic treatment on pain in adult patients with cysticfibrosis-A pilotrandomized controlled study. PLoS ONE 9(7):e102465

16. Koh JL, Harrison D et al (2005) Assessment of acute and chronic pain symptoms in children with cystic fibrosis. Pediatr Pulmonol 40(4):330-335

17. Kötter T, da Costa BR et al (2015) Metamizoleassociated adverse events: a systematic review and meta-analysis. PLoS ONE 10(4):e122918

18. Littlewood JM (1995) Abdominal pain in cystic fibrosis. JR Soc Med 88(Suppl 25):9-17

19. Maier C, Kindler D et al (2017) Medikamentöse Schmerztherapie. In: Maier C, Diener HC, Bingel U (Hrsg) Schmerzmedizin, 5. Aufl. Urban \& Fischer, München, S398-483

20. McNamara C, Johnson M et al (2016) Yoga therapy in children with cysticfibrosis decreases immediate anxiety and joint pain. Evid Based Complement Alternat Med 2016:9429504

21. https://muko-berlin-brandenburg.de/mukoviszi dose-arthritis/.Zugegriffen: 8. März 2021

22. Nash EF, Ohri CM et al (2014) Abdominal pain in adults with cystic fibrosis. Eur J Gastroenterol Hepatol 26(2):129-136 
23. Ng SM, Moore HS (2016) Drug therapies for reducing gastric acidity in people with cystic fibrosis. Cochrane Database Syst Rev 2016(8):CD3424

24. Petzke F, Karst M et al (2019) Cannabis in der Medizin - Ein Positionspapier zu medizinischem Cannabis und cannabisbasierten Medikamenten in der Schmerzmedizin. Schmerz 33(2019):449-465 (Adhoc-Kommission der Deutschen Schmerzgesellschaft)

25. PloessI C, Pettit RS, Donaldson J (2014) Prevalence of depression and antidepressant therapy use in a pediatric cystic fibrosis population. Ann Pharmacother 48(4):488-493

26. Porst M, Wengler A et al (2020) Migräne und Spannungskopfschmerzen in Deutschland. J Health Monit. https://doi.org/10.25646/6988

27. Raspe H (2012) Rückenschmerzen. Gesundheitsberichtserstattung des Bundes, Bd. 53. Robert Koch-Institut, Berlin

28. Sandy NS, Massabki LHP et al (2020) Distal intestinal obstruction syndrome: a diagnostic and therapeutic challenge in cystic fibrosis. J Pediatr (Rio J) 96(6):732-740

29. Sermet-Gaudelus I, De Villartay P et al (2009) Pain in children and adults with cystic fibrosis: a comparative study. J Pain Symptom Manage 38(2):281-290

30. Stephen MJ, Chowdhury J et al (2020) Use of medical marijuana in cystic fibrosis patients. BMC Complement Med Ther 20(1):323

31. Stephens SE, Rigden SP (2002) Cystic fibrosis and renal disease. Paediatr Respir Rev 3(2):135-138

32. Trandafir LM, Leon MM et al (2019) Current practices and potential nanotechnology perspectives for pain related to cystic fibrosis. JClin Med 8(7):1023

33. Treede RD, Rief $W$ et al (2019) Chronic pain as a symptom or a disease. Pain 160(1):19-27

34. Vijayvargiya P, Camilleri M et al (2020) Systematic review with meta-analysis: efficacy and safety of treatments for opioid-induced constipation. Aliment Pharmacol Ther 52(1):37-53

35. Wigger D, Stange M (2017) Medikamente in der Pädiatrie - Inklusive Neonatologie/ Intensivmedizin, 5. Aufl. Urban \&Fischer, München, S4-216

36. Wilke BU, Kummer KK et al (2020) Chloride-The underrated lon in nociceptors. Front Neurosci 14:287

37. Yaoli Y, Trina $\mathrm{H}$ et al (2020) Attitudes of pain and opioids prescription practices in U.S. cystic fibrosis centers. J Cyst Fibros 11:S1569-1993(20)30814-6

38. Zernikow B (2021) Chronische Schmerzen bei Kindern und Jugendlichen - Die stille Epidemie. Der Schmerz. Editorial. Schmerz 2021(35):80-82

\section{Pain in cystic fibrosis-An underestimated problem. A narrative review}

Background: Cystic fibrosis (CF) is one of the most common inherited metabolic diseases in Germany. It affects various organ systems and often leads to pain, which is usually underestimated. To date, there are no guidelines on this relevant topic in German-speaking countries.

Methods: A systematic literature search was performed in PubMed, German language guidelines and literature on the topic of $C F$ and pain. Papers containing data on $C F$, on pain or its treatment in CF were considered relevant. The search retrieved a total of 1690 articles of which 41 were included in the present review

Results: The research revealed that pain of various kinds due to diverse organ involvement is widespread; however, no German language guidelines or consensus papers exist for the diagnostics and treatment of pain in CF. European and American studies reflect the great relevance and range of the topic but they do not contain data on the effectiveness of pain treatment. In addition to classical pain medication with analgesics, many patients also rely on alternative healing methods such as physical therapy, acupuncture or tetrahydrocannabinol (THC). In view of the multiple organ dysfunctions, the use of analgesics is associated with particular risks.

Conclusion: Pain treatment is commonly required in patients with cystic fibrosis and multiorgan involvement and has to be adapted to the progression of the disease. Contraindications and side effects of analgesics have to be considered on an individual basis. The inclusion of pain management in German language CF guidelines is urgently needed.

Keywords

Chronic disease · Complications · Therapy · Guideline · Side effects 